Research Article

\title{
Benefit Effect of Dendrobium officinale Ultrafine Powder on DSS- Induced Ulcerative Colitis Rats by Improving Colon Mucosal Barrier
}

\author{
Xiang Zheng $\mathbb{D}^{1},{ }^{1}$ Tao-Xiu Xiong $\mathbb{D}^{1},{ }^{1}$ Ke Zhang $\mathbb{D},{ }^{1}$ Fu-Chen Zhou ${ }^{\mathbb{D}},{ }^{1}$ Hui-Ying Wang $\mathbb{D}^{1}{ }^{1}$ \\ Bo Li $\mathbb{D}^{1},{ }^{1,2}$ Ying-Jie Dong, ${ }^{1}$ Xinglishang He, ${ }^{1}$ Lin-Zi Li, ${ }^{1}$ Qiao-Xian Yu $\mathbb{D}^{1},{ }^{3}$ Gui-Yuan Lv $\mathbb{D},{ }^{4}$ \\ and Su-Hong Chen ${ }^{1,2}$ \\ ${ }^{1}$ Zhejiang University of Technology, Hangzhou 310014, Zhejiang, China \\ ${ }^{2}$ Zhejiang Synergetic Traditional Chinese Medicine Research and Development Co., Ltd., Deqing 322099, Zhejiang, China \\ ${ }^{3}$ Zhejiang Senyu Co., Ltd., Yiwu 322099, Zhejiang, China \\ ${ }^{4}$ Zhejiang Chinese Medical University, Hangzhou 310053, Zhejiang, China
}

Correspondence should be addressed to Qiao-Xian Yu; sstpfd@126.com, Gui-Yuan Lv; zjtcmlgy@163.com, and Su-Hong Chen; chensuhong@zjut.edu.cn

Received 24 April 2021; Revised 26 September 2021; Accepted 9 October 2021; Published 30 December 2021

Academic Editor: Xiaolong Ji

Copyright (C) 2021 Xiang Zheng et al. This is an open access article distributed under the Creative Commons Attribution License, which permits unrestricted use, distribution, and reproduction in any medium, provided the original work is properly cited.

\begin{abstract}
Aim and Objective. To study the effect of Dendrobium officinale ultrafine powder (DOFP) on the intestinal mucosal barrier in rats with ulcerative colitis (UC) induced by dextran sulfate sodium (DSS). Materials and Methods. After intragastric administration of DOFP for 3 weeks, the rat UC model was made by the administration of $4 \%$ oral DSS solution for one week, and the drug was given at the same time. During the experiment, the disease activity index (DAI) score of the rats was regularly computed. At the end of the experiment, the blood routine indexes of rats were obtained. The histopathological changes in the colon were monitored by hematoxylin-eosin (H\&E) and PAS staining and observation of ultrastructural changes in the colon by transmission electron microscope. Occludin expression in the colon was monitored by Western blot, the expression of claudin- 1 and ZO- 1 in the colon was detected by immunofluorescence, and the expression of TNF- $\alpha$, IL- 6 , and IL- $1 \beta$ in the colon was detected by immunohistochemistry. Results. The results firstly indicated that DOFP could significantly alleviate the signs and symptoms of the DSSinduced rats UC model, which manifested as improvement of body weight loss, increase of colon length, and improvement of the symptoms of diarrhea and hematochezia. Then, results from histopathology, blood routine examination, and transmission electron microscope analysis further implied that DOFP could dramatically reduce inflammatory cell infiltration and restore intestinal epithelial barrier integrity. In addition, the experiments of Western Blot analysis, immunofluorescence, and PAS staining also further confirmed that DOFP could markedly increase related protein expressions of the intestinal barrier and mucus barrier, as the expression of occludin, claudin-1, and ZO-1 in the colon significantly decreased. The experiments of immunohistochemistry confirmed that DOFP could markedly decrease protein expression levels of inflammatory cytokines TNF- $\alpha$, IL6 , and IL-1 $\beta$. Conclusion. DOFP notably alleviated inflammatory lesions, repaired the colon mucosa damage by promoting the expression of tight junction proteins occludin, claudin-1, and ZO-1 and inhibiting the release of inflammatory factors TNF- $\alpha$, IL6 , and IL- $1 \beta$, and finally achieved the purpose of treating UC.
\end{abstract}

\section{Introduction}

Ulcerative colitis (UC) is an inflammatory bowel disease characterized by inflammatory cell infiltration into the colonic mucosa. The etiology and pathogenesis of ulcerative colitis are not clear $[1,2]$. This disorder severely affects the normal life of patients and also increases the risk of secondary infections and colon cancer [3]. At present, the drugs used to treat UC are corticosteroids, 5-aminosalicylic acid (5-ASA), immunosuppressants, and biological agents or 
their derivatives [4]. However, the side effects of these drugs have a negative impact upon the liver, as well as psychological functions and other body systems [5]. Therefore, there is an urgent need to obtain therapeutic drugs with definite curative effects and few side effects.

Although the exact pathophysiological mechanism of UC is not clear, studies suggest that diet, environment, and intestinal mucosal injury are closely related to the occurrence of UC [6-8]. Tight junctions (TJs) mainly include transmembrane proteins, occludins, claudins, cytoplasmic, and attachment proteins of the ZO family proteins. In-depth studies have found that the expression of TJ protein is abnormal in UC, and this directly affects the intestinal mucosal barrier function and plays a key role in promoting the occurrence and development of UC [9]. Intestinal epithelial TJ protein can not only block the abnormal immune response caused by pathogenic intestinal microorganisms but also alleviate the inflammatory response caused by excessive leakage of bacteria and antigens through the mucous membrane [8]. Therefore, when there is a decrease in TJs, intestinal permeability increases, and this can cause celiac disease, diabetes, UC, and other diseases [10].

Dendrobium officinale Kimura et Migo (D. officinale) is a plant that has the effect of "benefiting the stomach and promoting fluid" [11], and its uses were recorded in the Shennong Materia Medica over 2000 years ago. The dried stem is the plant part of $D$. officinale that is medicinally used, and modern pharmacological studies have shown that D. officinale has a variety of biological activities, such as hepatoprotective, anticancer, hypoglycemic, antifatigue, and gastric ulcer protection [12]. D. officinale has a variety of active principles, such as polysaccharides, flavonoids, alkaloids, and essential oils. And polysaccharides are the dominant substances [13]. It has been reported that D. officinale and its polysaccharides have many beneficial effects on UC, such as decreasing colonic inflammation and regulating intestinal flora [14-16]. Ultrafine grinding technology uses mechanical or hydrodynamic methods to crush raw materials into a nanopowder or micropowder. After ultrafine pulverization, the particle size of a drug can reach $1-10 \mu \mathrm{m}$ as an ultrafine powder [17]. The powder has the advantages of convenient transportation and storage, excellent water solubility, low loss of functional components, high utilization rate, and beneficial dietary fiber $[18,19]$.

Previous laboratory studies have shown that D. officinale ultrafine powder (DOFP) could increase the expression of intestinal ZO-1 in convalescent nonalcoholic fatty liver model mice and regulate the abnormality of the intestinal-liver axis by inhibiting LPS-TLR4-related inflammation [18]. However, whether D. officinale can inhibit intestinal inflammation by regulating TJ protein in the UC model requires further study. We speculated that DOFP might act on the TJ proteins of the intestinal mucosal barrier to subsequently ameliorate UC. Therefore, in this study, dextran sulfate sodium (DSS) was used to induce UC in rats, and the focus was on the TJ proteins occludin, claudin-1, and ZO-1 to explore the efficacy and mechanism of DOFP in the prevention and treatment of ulcerative colitis.

\section{Materials and Methods}

2.1. Materials and Reagents. DSS (relative molecular weight 40000, Hubei Xinghengkang Chemical Technology Co., Ltd.) and the reagents of hematoxylin and eosin were purchased from the Institute of Biological Engineering of Nanjing Jiancheng Co. Ltd. (Nanjing, China).

2.2. Animal Treatment. Forty healthy male SD rats $(200 \pm 20$ g) were purchased from the Zhejiang Experimental Animal Center (Hangzhou, China), license number: SCXK (Zhejiang) 2014-0001. All the animals were housed at room temperature of $25^{\circ} \mathrm{C}$ and at $45-55 \%$ relative humidity with a 12-hour light-dark cycle. All rats were fed a standard pellet chow diet, and the gastric volume was $1 \mathrm{~mL} / 100 \mathrm{~g}$. All animal experiments were conducted with the approval of the Animal Care and Use Committee of Zhejiang University of Technology (ethical approval number: 20200603038).

2.3. DOFP Preparation. Dried D. officinale was purchased from Zhejiang Senyu Co., Ltd. (Zhejiang, China). The dried herb was crushed to create $D$. officinale coarse powder (DOFC) and D. officinale ultrafine powder (DOFP). The DOFP and DOFC were observed by scanning electron microscope (JEOL JSM-IT100, Beijing, China), the samples were fixed on scanning electron microscopy stubs using double-sided adhesive tape and then coated with $\mathrm{Au}$ at $50 \mathrm{~mA}$ for $3 \mathrm{~min}$ through a sputter coater, and a scanning electron microscope with a secondary electron detector was used to obtain digital images of the samples at an accelerating voltage of $20 \mathrm{kV}$.

The content of polysaccharides was determined by the phenol sulfuric acid method, and the maximum absorbance was found at $488 \mathrm{~nm}$ [18]. With standard glucose (Shanghai Yuanye Bio-Technology Co., Ltd., Shanghai, China) as the control substance, $1 \mathrm{~mL}$ extract sample or glucose standard solution was added to a $10 \mathrm{~mL}$ plugged test tube, and water was added to $1 \mathrm{~mL}$, mixed with $1 \mathrm{~mL} 5 \%$ phenol (Shanghai Yuanye Bio-Technology Co., Ltd., Shanghai, China), then mixed with $5 \mathrm{~mL}$ sulfuric acid (Sinopharm Chemical Reagent Co., Ltd, Shanghai, China), reacted in a boiling water bath for $20 \mathrm{~min}$, and cooled in an ice bath for $5 \mathrm{~min}$ to stop the reaction. The absorbance was measured at $488 \mathrm{~nm}$. The standard regression equation for glucose is $y=0.0364 x-0.0027 ; R=0.9984$ ( $y$ denotes absorbance, while $x$ denotes the concentration of glucose).

2.4. Animal Groups. Rats were randomly divided into four groups, the normal group (NG), model group (MG), DOFP low-dose group (DOFP-L, at a dose of $0.3 \mathrm{~g} / \mathrm{kg}$ ), and DOFP high-dose group (DOFP-H, at a dose of $0.6 \mathrm{~g} / \mathrm{kg}$ ), with ten animals in each group. The DOFP-L and DOFP-H rats received the corresponding drugs on a daily basis (p.o.) for 4 weeks. After 3 weeks of treatment, except for the NG, 4\% DSS solution was added to all other groups of rats drinking water ad libitum to induce ulcerative colitis (UC). For the 4 weeks experiment, all rats were fed with the standard diet, 
TABLE 1: Scoring standard of the disease activity index (DAI) [20].

\begin{tabular}{lccc}
\hline Score & Body weight decrease rate (\%) & Fecal property & Hematochezia status \\
\hline 0 & 0 & Normal & Normal \\
1 & $1-5$ & Semiloose $(+)$ & Feces with occult blood $(+)$ \\
2 & $6-10$ & Semiloose $(++)$ & Feces with occult blood $(++)$ \\
3 & $11-15$ & Loose $(+)$ & Bloody feces $(+)$ \\
4 & $>15$ & Loose $(++)$ & Bloody feces $(++)$ \\
\hline
\end{tabular}

and the rats in the NG and MG were provided with water every day (p.o.). Referring to the Pharmacopoeia of the People's Republic of China (2020 Edition), the recommended daily dosage of Dendrobium officinale is 6-12 g. The administration dosage for the rats was converted according to the surface area of a $60 \mathrm{~kg}$ human body, the high-dose Dendrobium officinale ultrafine powder is $0.6 \mathrm{~g} / \mathrm{kg}$, and the low dose is $0.3 \mathrm{~g} / \mathrm{kg}$ in this study. In addition, previous laboratory studies have shown that the $0.6 \mathrm{~g} / \mathrm{kg}$ dose group of Dendrobium officinale ultrafine powder can increase the expression of tight junction protein in the intestine of nonalcoholic fatty liver model mice during the recovery period, inhibit LPS-TLR4 related inflammation, and regulate the abnormality of the intestinal-liver axis [18].

2.5. Disease Activity Score. After the establishment of the model, the body weight, bleeding, and fecal consistency of the rats were measured and recorded every day (Table 1) [20].

2.6. Blood Routine Index Detection. On the last day of the experiment, 12 hours after fasting, retroorbital blood was sampled and placed in an EP tube pretreated with Ethylene Diamine Tetraacetic Acid (EDTA) to prepare whole blood. The white blood corpuscles (WBC), red blood corpuscles (RBC), hemoglobin (HGB), hematocrit (HCT), mean cell volume (MCV), mean corpuscular hemoglobin $(\mathrm{MCH})$, mean erythrocyte hemoglobin concentration (MCHC), platelet count (PLT), mean platelet volume (MPV), and eosinophil (EO) counts were analyzed using an automatic hematology analyzer (Sysmex Corporation, XT-2000i, Shanghai, China).

\subsection{Colonic Length Measurement and Tissue Preservation.} After anesthetization with pentobarbital sodium (Chengdu Huaxia Chemical Reagent Co., Ltd., Sichuan, China), blood samples were removed from the abdominal aorta of rats, then the colon was dissected and quickly separated, and the length of colon was measured. Part of the colon was stored at $-80^{\circ} \mathrm{C}$ for Western blot examination; another part was cut into small pieces and fixed in $2.5 \%$ glutaraldehyde solution to be observed by transmission electron microscope; and the remainder was fixed in $4 \%$ neutral formalin for $\mathrm{H} \& \mathrm{E}$ staining, PAS staining, and immunofluorescence analysis.

2.8. Colon Tissue H\&E Staining and Histopathological Score. The fixed colon tissue was embedded in paraffin after dehydration, and $4 \mu \mathrm{m}$ paraffin sections were prepared. After dewaxing and hydration, $\mathrm{H} \& \mathrm{E}$ staining was performed. The paraffin sections were observed and photographed by optical biological microscope and scored according to a standard procedure (Table 2) [21].

2.9. PAS Staining. For staining, $4 \mu \mathrm{m}$ paraffin sections were prepared. After dewaxing and hydration, the samples were treated with periodic acid dye, and incubation was performed for $6.5 \mathrm{~min}$. After rinsing with distilled water, the samples were treated with Schiff reagent and were incubated away from light for $15 \mathrm{~min}$ and then rinsed with running water. Then, hematoxylin staining was performed for $1.5 \mathrm{~min}$, and the samples were subsequently rinsed with running water and then dried. The tissues were observed, and images were recorded with an optical biological microscope.

2.10. Ultrastructural Observation of Colon in UC Rats. For structural examination, 2-3 pieces of colon tissue were submerged in $2.5 \%$ glutaraldehyde fixative solution for more than 24 hours. Then, ultrathin sections were created, and the ultrastructure of colonic epithelial cells was observed by transmission electron microscope (HITACHI, HT7700, $80 \mathrm{kV}$, China).

2.11. Western Blot Analysis. Liquid nitrogen was used for the mechanical lysis of 50-100 mg frozen colon tissues. Then, the tissues were lysed in RAPI buffer with protease/phosphatase inhibitor for $30 \mathrm{~min}$ on ice. The lysates were clarified by centrifugation at $10,000 \mathrm{rpm}$ for $20 \mathrm{~min}$ at $4^{\circ} \mathrm{C}$, and the protein concentrations were determined using BCA protein analysis kits (Shanghai Beyotime Biotechnology Co., Ltd., Shanghai, China). After adjusting the concentration, electrophoretic separation and membrane transfer were carried out. The membrane was incubated in 5\% milk at room temperature for 2 hours, and the first antibody of occludin or GAPDH (Proteintech Group, Shanghai, China) was incubated with the membrane overnight at $4^{\circ} \mathrm{C}$. After three washes in TBST for $5 \mathrm{~min}$ each, the second antibody was incubated with the membrane at room temperature for 2 hours. After rinsing with TBST, the bands were identified by chemiluminescence, and the gray value of the corresponding protein band was analyzed by ImageJ software.

2.12. Immunofluorescence. For immunofluorescence staining, $4 \mu \mathrm{m}$ paraffin sections were prepared, and after dewaxing and hydration, the samples were incubated in 3\% $\mathrm{H}_{2} \mathrm{O}_{2}$ for $15 \mathrm{~min}$ at room temperature. After three washes in 
TABLe 2: Histological scoring system [21].

\begin{tabular}{lcccc}
\hline Score & Inflammation & Mucosal damage & Crypt damage & Range of lesions (\%) \\
\hline 0 & None & None & None & 0 \\
1 & Mild & Mucous layer & $1 / 3$ & $1-25$ \\
2 & Moderate & Submucosa & $2 / 3$ & $26-50$ \\
3 & Severe & Muscularis and serosa & $100 \%$ & $51-75$ \\
4 & - & - & $100 \%+$ epithelium & $76-100$ \\
\hline
\end{tabular}

PBS for 5 min each, the samples were incubated in 5\% BSA at room temperature for 2 hours. The first antibody of claudin1 and ZO- 1 was incubated with the sections overnight at $4^{\circ} \mathrm{C}$. The second fluorescent antibody was incubated with the sections at room temperature for $30 \mathrm{~min}$. After rinsing with PBS and then staining with DAPI, the paraffin sections were observed and photographed under a fluorescence microscope (Olympus BX43, China).

2.13. Immunohistochemistry. $4 \mu \mathrm{m}$ paraffin sections were used for immunohistochemistry, and following the elimination of the endogenous peroxidase activity and antigen reparation, the tissues were sealed with blocking buffer. Then, the tissues were incubated with anti-TNF- $\alpha$, anti-IL-6, and anti-IL-1 $\beta$ (Proteintech Group, Shanghai, China) overnight at $4^{\circ} \mathrm{C}$ and subsequently with the secondary antibody. The DAB chromogen was used for incubation, and then hematoxylin was used for counterstaining. After dehydration of gradient ethanol and sealing slices with neutral gum, slices were observed under a microscope. The positive results were stained in yellow. The images were analyzed using Image-Pro Plus software.

2.14. Statistical Method. All the data were statistically analyzed by SPSS 22 software, and the data were expressed as mean \pm SD. The comparison between groups was performed by single-factor analysis of variance (ANOVA).

\section{Results}

3.1. Quality Control of DOFP. The DOFP and DOFC were observed and photographed under a scanning electron microscope. There were many intact cells in the DOFC (Figure 1(b)), while DOFP (Figure 1(a)) had fewer intact cells, and most of them were cell fragments. DOFC and DOFP contained $46.50 \%$ and $56.67 \%$ polysaccharides, respectively, as determined by the phenol sulfuric acid method, which indicated that there was greater polysaccharide dissolution of DOFP as compared to DOFC.

3.2. DOFP Relieved the Signs and Symptoms of UC in Rats Induced by DSS. The rats in each group showed different degrees of colitis via symptoms such as weight loss, diarrhea, and hematochezia. Compared with the normal group, the length of colon in the model group was shorter (Figures 2(a) and $2(\mathrm{~b})$ ), and the disease activity index (DAI) scores were significantly increased $(P<0.05)$ (Figure $2(c)$ ), suggesting that the model of colitis induced by DSS was successful.
Compared with the model group, both high and low doses of DOFP improved the symptoms of colon shortening in model rats, and it significantly improved the DAI score in model rats $(P<0.05)$. The above data indicated that DOFP treatment improved DSS-induced colitis in rats.

3.3. The Effect of DOFP on Routine Blood Indexes of DSSInduced Colitis Model Rats. The results of the blood routine examination of rats showed that WBC, HGB, and PLT in the model group were significantly higher than those in the normal group $(P<0.05)$, and MCV of the model group was significantly lower than that in the normal group (Table 3). Compared with the model group, the levels of WBC, HGB, and PLT in the DOFP-H group were significantly decreased $(P<0.05)$, while the MCV in the DOFP-L and DOFP-H groups was significantly increased $(P<0.05)$. It is suggested that DOFP can reduce WBC and other inflammatory indexes and decrease inflammatory lesions in UC model rats.

\subsection{The Effect of DOFP on the Pathological Changes in Colon} Tissue in DSS-Induced Colitis Model Rats. The results of H\&E staining showed that, compared with the normal group, the colonic mucosa of the model group was severely damaged, a large number of inflammatory cells infiltrated, and the crypt and glandular structures were severely damaged. Compared with the model group, the DOFP-L and DOFP-H groups exhibited decreased colonic mucosal injury, inflammatory cell infiltration, and crypt and glandular structure destruction and other pathological changes of the model rats (Figure 3(a)). The histopathological score of the model group was significantly higher than that of the normal group $(P<0.01)$, and the histopathological score of the DOFP-L and DOFP-H groups was significantly lower than that of the model group $(P<0.05,0.01)$ (Figure $3(\mathrm{~b}))$.

\subsection{The Effect of DOFP on Goblet Cells and Mucus in the Colons} of DSS-Induced Colitis Model Rats. The results of PAS staining showed that the shape of colonic goblet cells in the normal group was full and round, mucus secretion was abundant, and the mucosal surface was covered with mixed mucus. Compared with the normal group, the goblet cells in the model group atrophied to varying degrees, and the mucus cover on the mucous membrane was decreased. Compared with the model group, the atrophy of intestinal mucosal epithelial cells and goblet cells in the DOFP-L and DOFP-H groups was reversed by varying degrees, and the mucus secretion increased (Figure 3(c)). 


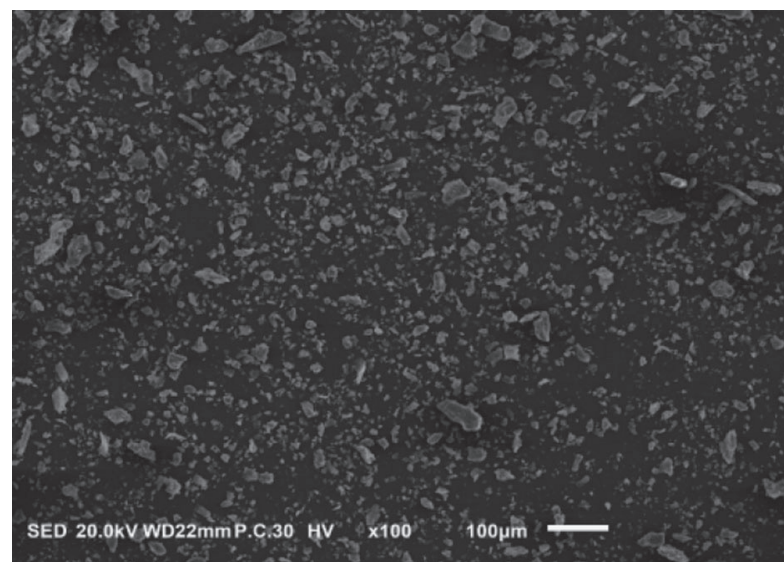

(a)

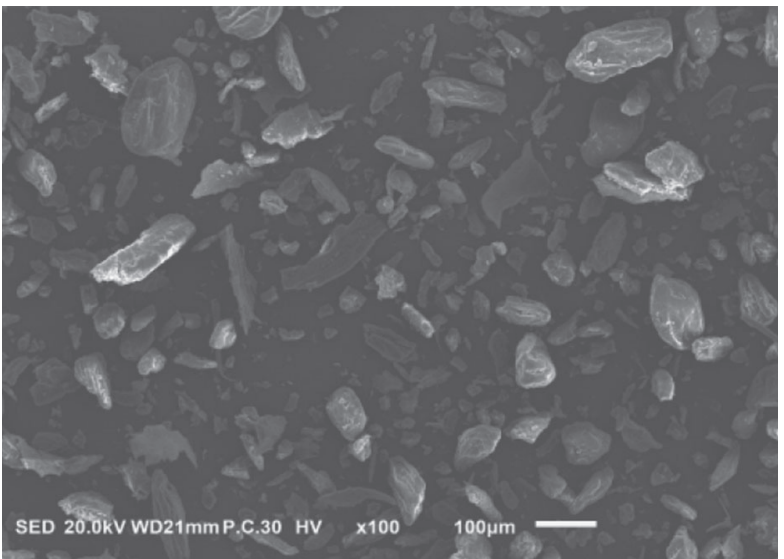

(b)

FIgURE 1: Characteristics of DOFC and DOFP. (a) SEM micrographs of DOFP. (b) SEM micrographs of DOFC.

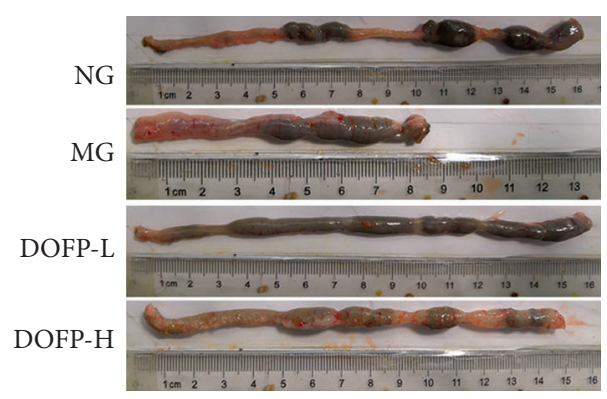

(a)

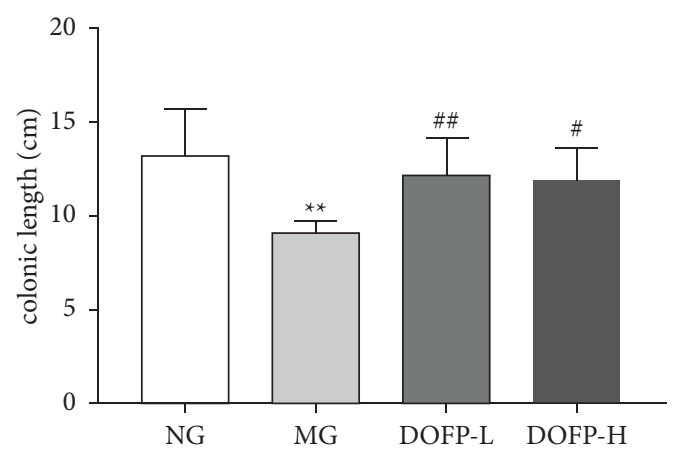

(b)

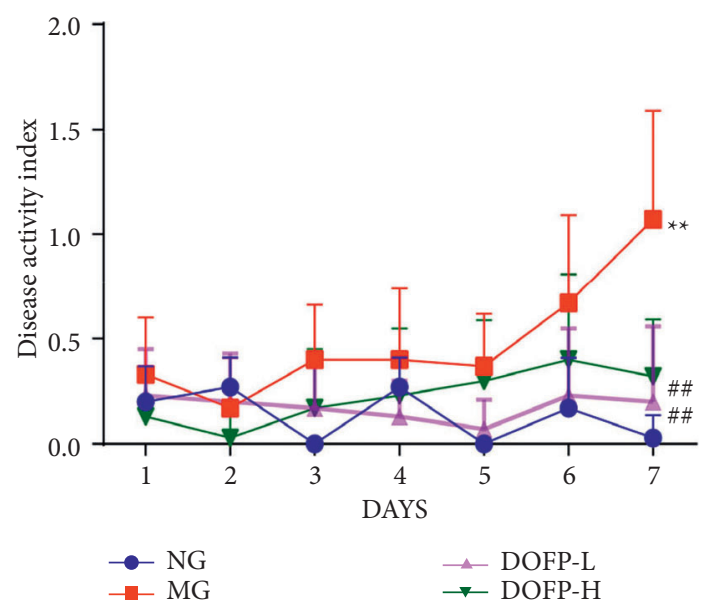

(c)

Figure 2: DOFP treatment improves DSS-induced colitis in rats. (a) Representative photos of colon length. (b) Colon length. (c) Disease activity score. ${ }^{* *} P<0.01$, compared with the normal group; ${ }^{\#} P<0.05$ and ${ }^{\# \#} P<0.01$, compared with the model group.

3.6. Transmission Electron Microscope Observation. The results of TEM showed that the microvilli on the surface of the colonic mucosa epithelial cells in the normal group were intact, the epithelial cells were tightly connected, and the intercellular space exhibited no obvious abnormal changes. In the model group, the rat colonic mucosal epithelial cells 
TABLE 3: Effect of DOFP on blood indexes of rats.

\begin{tabular}{lcccc}
\hline Project & NG & MG & DOFP-L & DOFP-H \\
\hline WBC $\left(10^{9} / \mathrm{L}\right)$ & $8.95 \pm 1.31$ & $12.35 \pm 3.49^{*}$ & $10.39 \pm 1.14$ & $9.87 \pm 1.82^{\#}$ \\
RBC $\left(10^{12} / \mathrm{L}\right)$ & $10.26 \pm 0.52$ & $10.80 \pm 0.90$ & $10.65 \pm 0.60$ & $10.13 \pm 0.80$ \\
HGB $(\mathrm{g} / \mathrm{L})$ & $185.11 \pm 6.25$ & $193.89 \pm 11.49^{*}$ & $194.70 \pm 9.93$ & $181.00 \pm 11.80^{\#}$ \\
HCT (\%) & $54.38 \pm 2.70$ & $56.70 \pm 3.23$ & $56.57 \pm 2.90$ & $54.41 \pm 3.30$ \\
MCV (Fl) & $54.63 \pm 2.92$ & $51.91 \pm 2.00^{*}$ & $53.80 \pm 1.55^{\#}$ & $54.32 \pm 2.17^{\#}$ \\
MCH (pg) & $18.23 \pm 0.87$ & $17.99 \pm 0.65$ & $18.29 \pm 0.64$ & $18.25 \pm 0.59$ \\
MCHC (g/L) & $343.80 \pm 6.66$ & $342.00 \pm 4.18$ & $344.20 \pm 5.25$ & $339.00 \pm 8.25$ \\
PLT (10 $/ \mathrm{L})$ & $853.00 \pm 153.72$ & $1011.57 \pm 123.19^{*}$ & $961.88 \pm 179.04$ & $837.86 \pm 214.65^{\#}$ \\
MPV (fL) & $7.61 \pm 0.31$ & $7.68 \pm 0.24$ & $7.44 \pm 0.34^{\#}$ & $7.55 \pm 0.42$ \\
EO (\%) & $0.10 \pm 0.02$ & $0.10 \pm 0.04$ & $0.11 \pm 0.05$ & $0.12 \pm 0.04$ \\
\hline
\end{tabular}

${ }^{*} P<0.05$ and ${ }^{* *} P<0.01$, compared with the normal group; ${ }^{\#} P<0.05$ and ${ }^{\# \#} P<0.01$, compared with the model group.

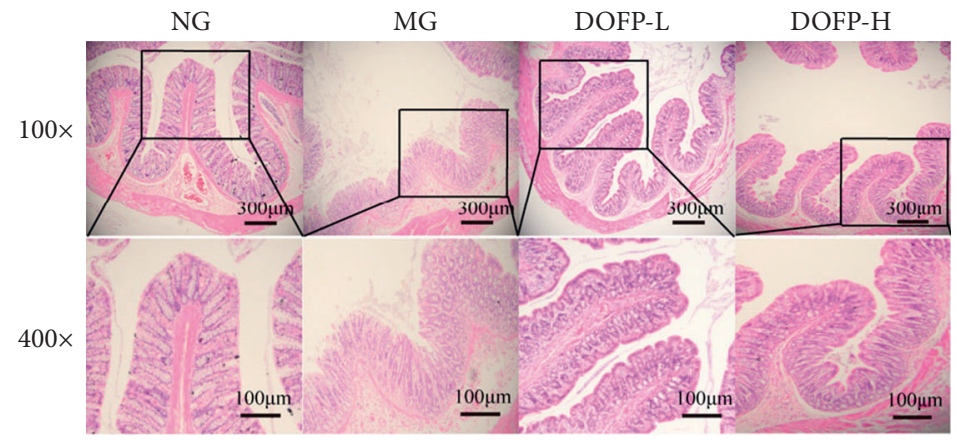

(a)

NG

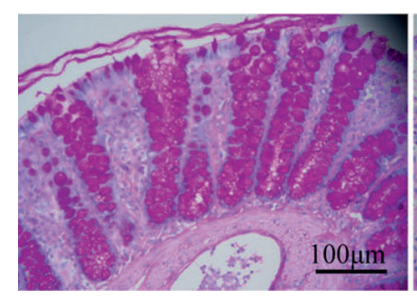

MG

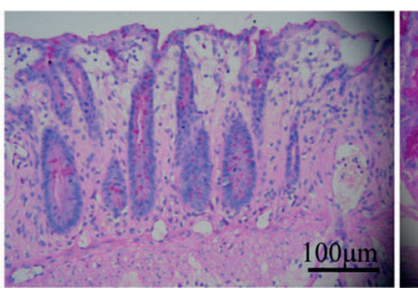

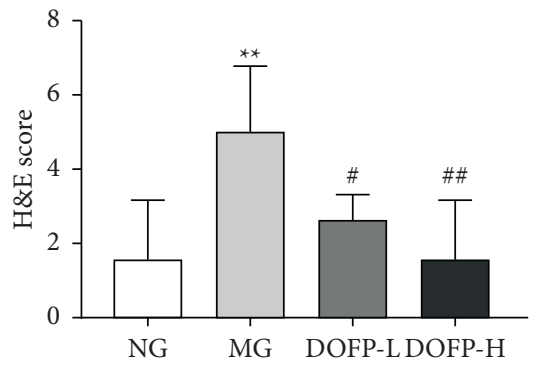

(b)

DOFP-L

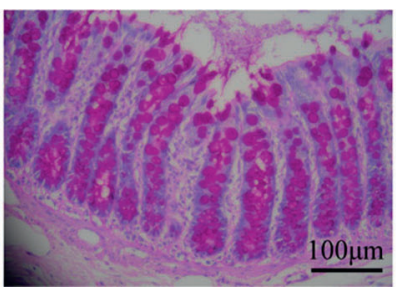

DOFP-H

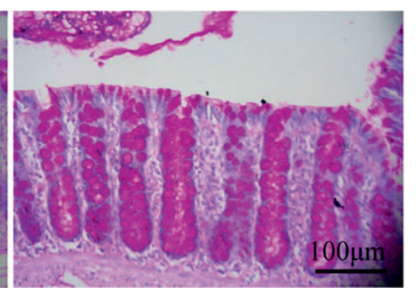

(c)

Figure 3: Effects of DOFP on histopathology of colitis rats. (a) Representative picture of colonic H\&E staining (100×, 400×). (b) Colonic $\mathrm{H} \& \mathrm{E}$ staining score. (c) Representative picture of colonic PAS staining $(400 \times) .{ }^{*} P<0.05$ and ${ }^{* *} P<0.01$, compared with the normal group; ${ }^{\#} P<0.05$ and ${ }^{\# \#} P<0.01$, compared with the model group.

lost microvilli, the intercellular space widened, the endoplasmic reticulum expanded, the mitochondria were swollen, part of the cytoplasm was dissolved, and apoptosis was visible. The colonic mucosal epithelial cells in rats from the DOFP group exhibited additional thick and short microvilli that were neatly arranged and uniform in size, the cells were neatly arranged, the degree of intercellular widening was significantly reduced, the glandular epithelia were closely connected to each other, and there was no expanded endoplasmic reticulum or swollen mitochondria (Figure 4(a)).

\subsection{The Effect of DOFP on the Expression of Occludin Protein in} Colon Tissue. Tight junction (TJ) protein plays an important role in maintaining a normal intestinal barrier. In order to clarify the effect of DOFP on TJ protein, the expression of occludin protein in the rat colon was detected by Western blot. The results showed that, compared with the normal group, the model group exhibited significantly decreased expression of occludin in colon tissue $(P<0.05)$. Compared with the model group, the DOFP-L group exhibited significantly increased expression of occludin protein in colon tissue $(P<0.05)$ (Figure $4(\mathrm{~b}))$.

3.8. The Effect of DOFP on the Expression of Claudin-1 and ZO1 Protein in Colon Tissue. The results of immunofluorescence showed that the expression of claudin-1 and ZO-1 protein in the colon tissue of the model group was lower than that of the normal group. Compared with the model group, the expression of claudin-1 and ZO-1 protein in the colon tissue of rats in the DOFP-L group increased (Figures 4(c) and 4(d)). It is suggested that the DOFP can increase the expression of occludin, claudin-1, ZO-1, and other tight junctions in the colon of UC model rats, and this subsequently acts to strengthen the intestinal mucosal barrier. 


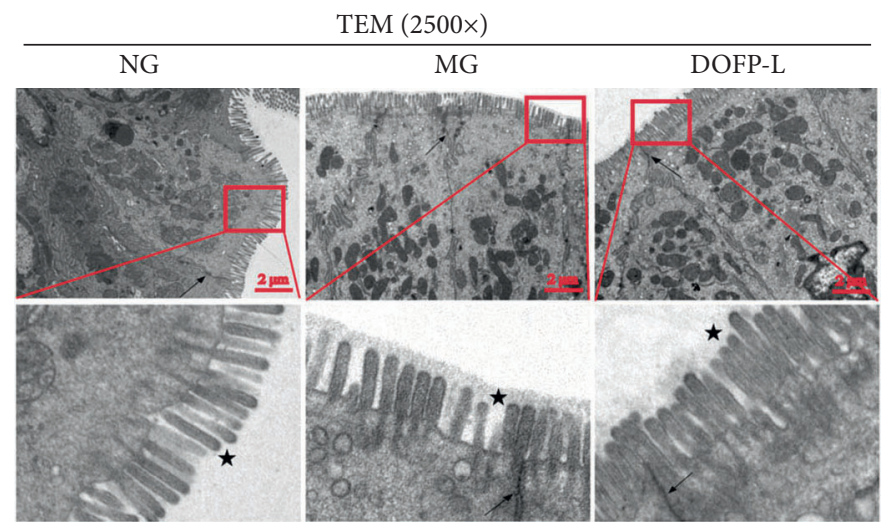

(a)

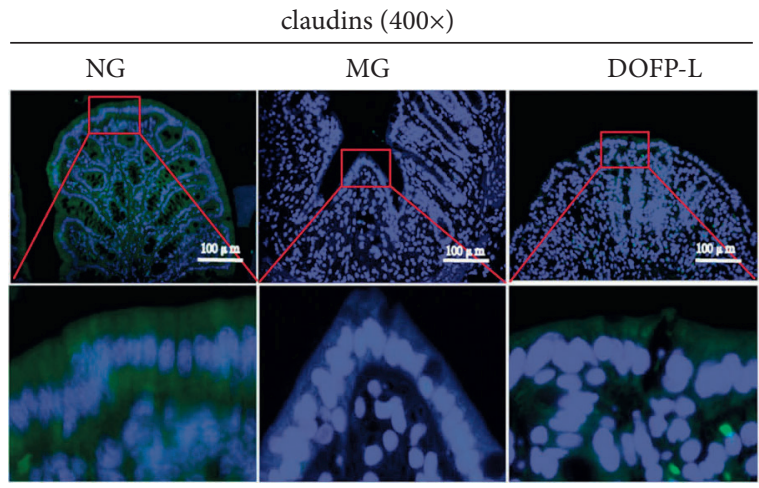

(c)

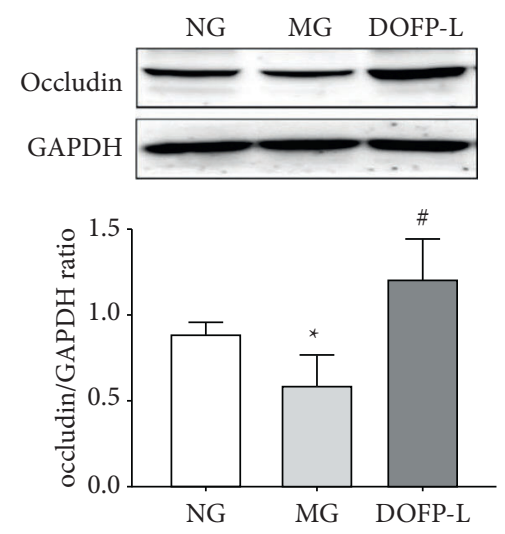

(b)

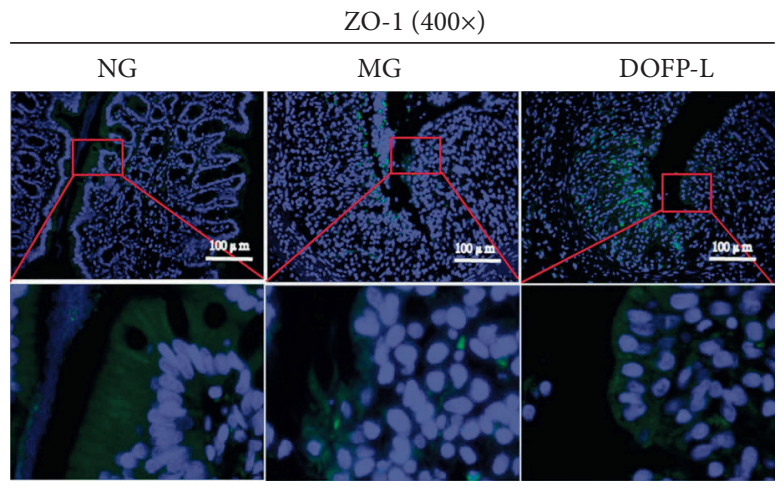

(d)

FIGURE 4: Effects of DOFP on TJ barrier function of colonic mucosa in UC rats. (a) Representative picture of the ultrastructure of colonic mucosa in colon. The arrow points to the tight junction, and the asterisk indicates microvilli. (b) Representative picture and semiquantitative statistical map of occludin protein bands in colon. (c) Representative picture of claudin-1 proteins expression with immunofluorescence in colon $(400 \times)$. (d) Representative picture of ZO-1 proteins expression with immunofluorescence in colon $(400 \times)$. ${ }^{*} P<0.05$ and ${ }^{* *} P<0.01$, compared with the normal group; ${ }^{\#} P<0.05$ and ${ }^{\# \#} P<0.01$, compared with the model group.

\subsection{The Effect of DOFP on the Expression of TNF- $\alpha, I L-6$, and} $I L-1 \beta$ Protein in Colon Tissue. The results of immunohistochemistry showed that the expression of TNF- $\alpha$, IL- 6 , and IL- $1 \beta$ protein in the colon tissue of the model group was higher than that of the normal group. Compared with the model group, the expression of TNF- $\alpha$, IL- 6 , and IL- $1 \beta$ protein in the colon tissue of rats in the DOFP-L group decreased (Figures 5(a) and 5(b)). It is suggested that the DOFP can decrease the expression of TNF- $\alpha$, IL- 6 , and IL- $1 \beta$ in the colon of UC model rats and play a role in inhibiting the intestinal inflammatory response.

\section{Discussion}

UC is a chronic inflammatory bowel disease, and its incidence has been increasing worldwide for many years [22]. Increasing evidence shows that the use of Chinese herbal medicine is effective for diseases, especially inflammatory diseases [23]. In the treatment of DSS-induced UC model mice with Lizhong decoction, colonic lesions were decreased in all dose groups, the disease activity index (DAI) score was decreased, and the expression of TJ proteins was upregulated [7]. Dendrobium officinale contains a variety of active ingredients, including polysaccharides, alkaloids, glycosides, phenanthrene, bibenzyl, and amino acids, among which polysaccharides have the highest content in D. officinale. Our previous literature showed that the total polysaccharide content $(47.96 \%, \mathrm{w} / \mathrm{w})$ and monosaccharide contents (consisted of D-mannose, glucose, galactose, and arabinose at $42.2 \%, 12.3 \%, 0.4 \%$, and $0.4 \%$, w/w) were detected in DOFP [18]. The total flavonoid content $(3.78 \pm 0.48 \%)$ and the content of naringenin $(0.3601 \pm 0.014 \mathrm{mg} / \mathrm{g})$ in DOFP were detected in a sodium hydroxide-aluminum sulfate method and high-performance liquid chromatograph (HPLC) system (1260, Agilent, Germany), respectively, as described in our previous report [19]. Modern pharmacological studies have shown that polysaccharides have anti-inflammatory, antioxidant, antitumor, and other pharmacological effects, enhance immunity, and regulate intestinal flora [24].

D. officinale has antifatigue, anti-inflammation, and antioxidation effects, reduces night sweats and dizziness, enhances immunity, and has been used in traditional Chinese medicine for more than 1000 years [23, 25]. D. officinale and its polysaccharides have many beneficial anti-UC effects, such as decreasing colonic inflammation and regulating intestinal flora $[14,15]$.

There are a large number of symbiotic bacteria in the colon. The intestinal mucosal barrier consists of closely 


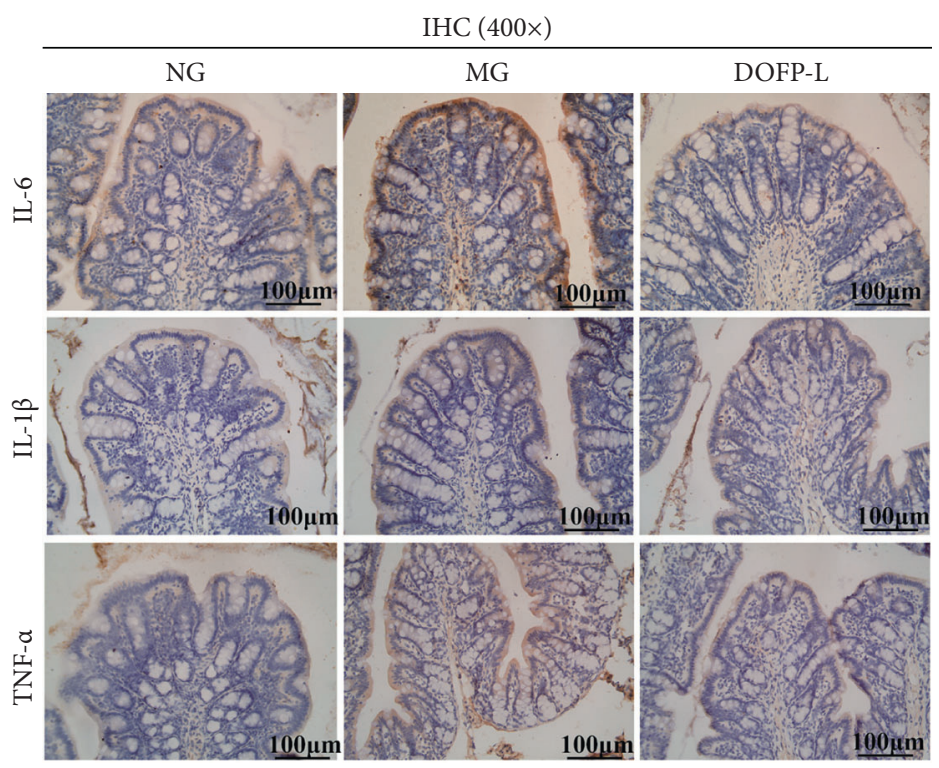

(a)
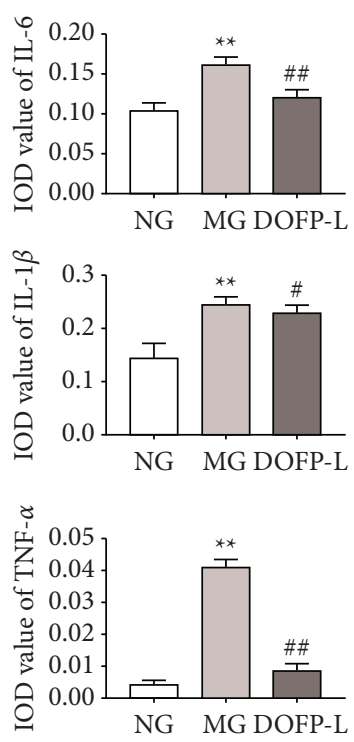

(b)

FIGURE 5: Effects of DOFP on the inflammatory factors TNF- $\alpha$, IL-6, and IL- $1 \beta$ of colonic mucosa in UC rats. (a) Representative picture of colonic IHC staining $(400 \times)$. (b) Colon IHC average optical density statistics chart. ${ }^{*} P<0.05$ and ${ }^{* *} P<0.01$, compared with the normal group; ${ }^{\#} P<0.05$ and ${ }^{\# \#} P<0.01$, compared with the model group.

arranged colonic epithelial cells that act as an important physical and immune obstacle between the intestinal flora and the body. It is the key factor for the body to maintain the stability of the intestinal environment [26]. Although the exact pathophysiological mechanism of UC is not clear, it has been proposed that the decrease in intestinal mucosal barrier permeability can cause severe inflammation and lead to the development of UC. Therefore, maintaining the integrity of the intestinal mucosal barrier is an important strategy for the treatment of UC [27]. Intestinal mucosal epithelial cells and their TJs form a complete epithelial barrier, which is an important part of the intestinal mucosal mechanical barrier [7].

The barrier function of the intestinal epithelium is partially maintained by the TJs between adjacent epithelial cells. The TJs are composed of a series of proteins with multiple functions, including occludins, claudins, and $\mathrm{ZO}$ family proteins. Studies have shown that downregulation of TJs may cause ectopic invasion of intestinal pathogens, antigens, and toxins through the colonic mucosal barrier, resulting in the activation of immune cells and abnormal immune response of the intestinal mucosa. This can destroy the mucosal barrier function and increase intestinal permeability, which can then promote the occurrence and development of UC [28].

Occludins protein is a direct component of the $\mathrm{TJ}$ protein structure, and because of its adhesion characteristics, it can form an intercellular barrier through multimerization. For example, with increasing levels of occludins protein, the intercellular adhesion increases, the transmembrane resistance increases, and the permeability of the intestinal mucosal barrier decreases [29]. Both claudins and occludins are cell membrane proteins that are the functional and structural basis of the TJ complex.

Small molecular transmembrane proteins with a relative molecular weight of approximately 22,000 can directly affect the transmembrane resistance of epithelial and endothelial tissues, determine the ion selectivity of the TJ complex, and regulate the TJ permeability [30]. ZO proteins are cytoplasmic proteins that contain PDZ domains, which contribute to the anchoring of $\mathrm{TJ}$ proteins such as claudins and occludins to the cytoplasm [31]. Therefore, occludins, claudins, ZO, and other family proteins are often used as indicators to evaluate $\mathrm{TJ}$ barrier function and permeability function in tissues and are also important indicators for monitoring the degree of intestinal mucosal barrier injury in UC.

In the current study, it was found that the expression of occludin, claudin-1, ZO-1, and other TJ proteins decreased in DSS-induced UC model rats, and the intestinal mucosal barrier was damaged. In a study on the effect of patchouli extract on DSS-induced UC model rats, the expression of occludin, claudin-1, ZO-1, and other TJ proteins decreased in the model group [23]. In the current study, we found that, after the intervention of DOFP, the protein expression of occludin, claudin-1, and ZO-1 in the colon of model rats was significantly increased, and the colonic mucosal barrier did not degrade. Some studies have shown that $D$. officinale polysaccharides can protect the intestinal mucosal barrier, reduce intestinal cell permeability, maintain intestinal balance, restore intestinal villus damage, and effectively improve intestinal barrier function by upregulating the expression of TJ proteins such as occludin, claudin-1, and ZO-1 and reducing apoptosis $[14,32]$. The previous study of 
our group showed that DOFP significantly increased the expression of occludin, claudin-1, and ZO-1 mRNA, tightened the loose arrangement of intestinal villi, protected the intestinal barrier function, and reduced the liver damage caused by lipopolysaccharide (LPS) in convalescent nonalcoholic fatty liver model mice (induced by a high-sugar and high-fat diet) [18]. DOFP repaired the damaged colonic mucosal barrier by upregulating the expression of occludin, claudin-1, and ZO-1TJ proteins.

DSS-induced UC model rats possess a disease pathology that is similar to that of human UC, including weight loss, diarrhea, hematochezia, intestinal mucosal injury, and other symptoms, in which the severity of hematochezia is one of the important indicators to evaluate the degree of mucosal barrier damage [5]. In the current study, the results of hematoxylineosin (H\&E) staining and periodic Acid-Schiff (PAS) staining showed that the colon of model rats sustained a mucosal injury, with inflammatory cell infiltration, an increase in goblet cells and mucus, the appearance of other histological lesions, and an increase in the DAI score. Some studies have shown that D. officinale polysaccharides can significantly reduce the DAI score of mice with UC induced by DSS and effectively ameliorate the hematochezia and weight loss $[14,33]$.

Mucosal barrier damage and inflammation are important pathological changes in the course of UC. DSS mainly develops intestinal inflammation by increasing intestinal permeability and damaging the intestinal barrier. It mainly affects the tight junction proteins of epithelial cells such as ZO-1 and occludin to damage the intestinal barrier, thereby increasing TNF- $\alpha$ and IL-6 expression of inflammatory factors [34].

In this study, we found that DOFP significantly reduced the DAI score of model rats, reduced the occurrence of hematochezia, reduced the shortening of the colon, improved the disappearance of microvilli of colonic mucosal epithelial cells and expansion of the endoplasmic reticulum, normalized the appearance of the mitochondria, upregulated the expression of TJ protein, downregulated the expression of inflammatory factors, effectively alleviated the injury of the intestinal mucosa, and improved the quality of life of the rats.

\section{Conclusions}

In a word, our results show that DOFP can improve DSSinduced colitis in model rats. It increases the expression of $\mathrm{TJ}$ proteins such as occludin, claudin-1, and ZO-1 in colon, inhibits the release of inflammatory factors TNF- $\alpha$, IL- 6 , and IL- $1 \beta$, repairs intestinal mucosal injury, reduces intestinal cell permeability, and maintain intestinal balance, so as to achieve the purpose of treating UC. These results suggest that $D$. officinale may be developed as a potential drug for the treatment of UC. However, D. officinale has a variety of active constituents, such as polysaccharides, flavonoids, alkaloids, and essential oils, which may be the possible active principle of this plant. We can further study it in the future.

5.1. Statement of Human and Animal Rights. All of the experimental procedures involving animals were conducted in accordance with the Institutional Animal Care guidelines of
Zhejiang University of Technology, China, and approved by the Animal Care and Use Committee of Zhejiang University of Technology, China.

\section{Data Availability}

The data to support the findings of this study are included within the article. Other data used to support the findings of this study are available from the corresponding author upon request.

\section{Ethical Approval}

All animal experiments were conducted with the approval of the Animal Care and Use Committee of Zhejiang University of Technology (ethical approval number: 20200603038).

\section{Conflicts of Interest}

The authors declare that they have no conflicts of interest.

\section{Authors' Contributions}

Xiang Zheng, Tao-Xiu Xiong, and Ke Zhang contributed equally to this paper.

\section{Acknowledgments}

This study was supported by the National Key Research and Development Program (no. 2017YFC1702200), the National Science Foundation of China (nos. 81874352, 81873036, 81673638, and 81803760), the Project of Zhejiang Research Institute of China Engineering Science and Technology Development Strategy (no. 2019-ZJ-JS-05), the Key Research and Development Program of Zhejiang Province (2017C03052), and the General Project Foundation of the Zhejiang Provincial Department of Education (Y202043163).

\section{References}

[1] H. Chu, X. Tao, Z. Sun, W. Hao, and X. Wei, "Galactooligosaccharides protects against DSS-induced murine colitis through regulating intestinal flora and inhibiting NF$\kappa$ B pathway," Life Sciences, vol. 242, Article ID 117220, 2020.

[2] Z. Lu, W. Xiong, S. Xiao et al., "Huanglian Jiedu Decoction ameliorates DSS-induced colitis in mice via the JAK2/STAT3 signalling pathway," Chinese Medicine, vol. 15, no. 1, 2020.

[3] Z. Yuan, L. Yang, X. Zhang, P. Ji, Y. Hua, and Y. Wei, "Huanglian-jie-du decoction ameliorates acute ulcerative colitis in mice via regulating NF- $\kappa \mathrm{B}$ and Nrf2 signaling pathways and enhancing intestinal barrier function," Frontiers in Pharmacology, vol. 10, no. 10, Article ID 1354, 2019.

[4] N. Jiang, Y. Wei, Y. Cen et al., "Andrographolide derivative al1 reduces intestinal permeability in dextran sulfate sodium (dss)-induced mice colitis model," Life Sciences, vol. 241, Article ID 117164, 2020.

[5] Z.-F. Zhang, H.-W. Zhao, X. Chai et al., "Effect of Hedyotis Diffusae Herba on dextran sulphate sodium-induced chronic ulcerative colitis in mice," Chinese Traditional and Herbal Drugs, vol. 46, no. 23, pp. 83-88, 2015. 
[6] Y. Sheng, T. Wu, Y. Dai, K. Ji, Y. Zhong, and Y. Xue, "The effect of 6-gingerol on inflammatory response and Th17/Treg balance in DSS-induced ulcerative colitis mice," Annals of Translational Medicine, vol. 8, no. 7, p. 442, 2020.

[7] Y. Shen, J. Zou, M. Chen et al., "Protective effects of Lizhong decoction on ulcerative colitis in mice by suppressing inflammation and ameliorating gut barrier," Journal of Ethnopharmacology, vol. 259, Article ID 112919, 2020.

[8] S. Huang, Y. Fu, B. Xu et al., "Wogonoside alleviates colitis by improving intestinal epithelial barrier function via the MLCK/ pMLC2 pathway," Phytomedicine, vol. 68, Article ID 153179, 2020.

[9] J. Wang, C. Zhang, C. Guo, and X. Li, "Chitosan ameliorates DSS-induced ulcerative colitis mice by enhancing intestinal barrier function and improving microflora," International Journal of Molecular Sciences, vol. 20, no. 22, Article ID 5751, 2019.

[10] S.-T. Wu, "Studies on chemical constituents and pharmacological effect of active fraction compound in treating ulcerative colitis in compound kushen decoction," Master thesis, Hubei University of Chinese Medicine, Wuhan, China, 2017.

[11] L. Gui-yuan, M.-Q. Yan, and S.-H. Chen, "Review of pharmacological activities of Dendrobium officinale based on traditional functions," China Journal of Chinese Materia Medica, vol. 38, no. 4, pp. 489-493, 2013.

[12] H. Zhao, X. Chen, X. Chen et al., "New peptidendrocins and anticancer chartreusin from an endophytic bacterium of Dendrobium officinale," Annals of Translational Medicine, vol. 8 , no. 7 , p. $455,2020$.

[13] H. Tang, T. Zhao, Y. Sheng, T. Zheng, L. Fu, and Y. Zhang, "Dendrobium officinale Kimura et Migo: a Review on Its Ethnopharmacology, Phytochemistry, Pharmacology, and Industrialization," Evidence-based Complementary and Alternative Medicine, vol. 2017, Article ID 7436259, 19 pages, 2017.

[14] J. Liang, H. Li, J. Chen et al., "Dendrobium officinale polysaccharides alleviate colon tumorigenesis via restoring intestinal barrier function and enhancing anti-tumor immune response," Pharmacological Research, vol. 148, Article ID 104417, 2019.

[15] L. Li, H. Yao, X. Li et al., "Destiny of Dendrobium officinale polysaccharide after oral administration: indigestible and nonabsorbing, ends in modulating gut microbiota," Journal of Agricultural and Food Chemistry, vol. 67, no. 21, pp. 59685977, 2019.

[16] Y. Yuan, J. Zhang, X. Liu, M. Meng, J. Wang, and J. Lin, "Tissue-specific transcriptome for Dendrobium officinale reveals genes involved in flavonoid biosynthesis," Genomics, vol. 112, no. 2, pp. 1781-1794, 2020.

[17] Y.-J. Yang, "Research progress on superfine grinding technique in field of Chinese materia medica," Chinese herbal medicine, vol. 50, no. 23, pp. 5887-5891, 2019.

[18] S.-S. Lei, B. Li, Y.-H. Chen et al., "Dendrobii Officinalis, a traditional Chinese edible and officinal plant, accelerates liver recovery by regulating the gut-liver axis in NAFLD mice," Journal of Functional Foods, vol. 61, Article ID 103458, 2019.

[19] S.-S. Lei, N.-Y. Zhang, F.-C. Zhou et al., "Dendrobium officinale regulates fatty acid metabolism to ameliorate liver lipid accumulation in NAFLD mice," Evidence-based Complementary and Alternative Medicine, vol. 2021, Article ID 6689727, 12 pages, 2021.

[20] Z. Liu, F. Liu, W. Wang et al., "Study of the alleviation effects of a combination of Lactobacillus rhamnosus and inulin on mice with colitis," Food \& Function, vol. 11, no. 5, pp. 3823-3837, 2020.

[21] Z. Zhang, S. Li, H. Cao et al., "The protective role of phloretin against dextran sulfate sodium-induced ulcerative colitis in mice," Food and Function, vol. 10, no. 1, pp. 422-431, 2019.

[22] F. Xie, H. Zhang, C. Zheng, and X.-f. Shen, "Costunolide improved dextran sulfate sodium-induced acute ulcerative colitis in mice through NF- $\kappa \mathrm{B}$, STAT1/3, and Akt signaling pathways," International Immunopharmacology, vol. 84, Article ID 106567, 2020.

[23] Z. Wu, H. Zeng, L. Zhang et al., "Patchouli alcohol: a natural sesquiterpene against both inflammation and intestinal barrier damage of ulcerative colitis," Inflammation, vol. 43, no. 4, pp. 1423-1435, 2020.

[24] X. Ji, C. Hou, Y. Gao, Y. Xue, Y. Yan, and X. Guo, "Metagenomic analysis of gut microbiota modulatory effects of jujube (Ziziphus jujuba Mill.) polysaccharides in a colorectal cancer mouse model," Food \& Function, vol. 11, no. 1, pp. 163-173, 2020.

[25] Z. Yu, Y. Liao, J. Teixeira da Silva, Z. Yang, and J. Duan, "Differential accumulation of anthocyanins in Dendrobium officinale stems with red and green peels," International Journal of Molecular Sciences, vol. 19, no. 10, Article ID 2857, 2018.

[26] S. Y. Salim and J. D. Söderholm, "Importance of disrupted intestinal barrier in inflammatory bowel diseases," Inflammatory Bowel Diseases, vol. 17, no. 1, pp. 362-381, 2011.

[27] H. Lee, Y. S. Son, M.-O. Lee et al., "Low-dose interleukin-2 alleviates dextran sodium sulfate-induced colitis in mice by recovering intestinal integrity and inhibiting AKT-dependent pathways," Theranostics, vol. 10, no. 11, pp. 5048-5063, 2020.

[28] S. Yin, H. Yang, Y. Tao et al., "Artesunate ameliorates DSSinduced ulcerative colitis by protecting intestinal barrier and inhibiting inflammatory response," Inflammation, vol. 43, no. 2, pp. 765-776, 2020.

[29] W. Fries, A. Belvedere, and S. Vetrano, "Sealing the broken barrier in IBD: intestinal permeability, epithelial cells and junctions," Current Drug Targets, vol. 14, no. 12, pp. 1460-1470, 2013.

[30] Y.-X. Rao, J. Chen, and Y.-D. Wu, "Changes in the expression of liver tight junction protein in dextran sulfate sodium induced ulcerative colitis rat model," Journal of pediatrics, vol. 30, no. 10, pp. 967-972, 2012.

[31] S. Shastri, T. Shinde, K. L. Woolley, J. A. Smith, N. Gueven, and R. Eri, "Short-chain naphthoquinone protects against both acute and spontaneous chronic murine colitis by alleviating inflammatory responses," Frontiers in Pharmacology, vol. 12, Article ID 709973, 2021.

[32] K. Wang, X. Yang, Z. Wu et al., "Dendrobium officinale polysaccharide protected CCl4-induced liver fibrosis through intestinal homeostasis and the LPS-TLR4-NF- $\kappa$ b signaling pathway," Frontiers in Pharmacology, vol. 11, Article ID 240, 2020.

[33] J. Liang, S. Chen, J. Chen et al., "Therapeutic roles of polysaccharides from Dendrobium Officinaleon colitis and its underlying mechanisms," Carbohydrate Polymers, vol. 185, pp. 159-168, 2018.

[34] C.-L. Zhu, J.-J. Mu, and J.-W. Xu, "Repair effect of purple sweet potato anthocyanin on intestinal barrier injury in mice with ulcerative colitis induced by DSS," Chinese Journal of Pathophysiology, vol. 36, no. 10, pp. 1844-1853, 2020. 